\title{
SHADING WITHOUT SHAPE
}

\author{
BY \\ MICHAEL J. BROOKS \\ University of Adelaide, Adelaide, Australia \\ WOJCIECH CHOJNACKI \\ University of Warsaw, Warsaw, Poland \\ Flinders University of South Australia, Adelaide, Australia \\ AND \\ RYSZARD KOZERA \\ University of Western Australia, Nedlands, Australia
}

\begin{abstract}
A smooth object depicted in a monochrome image will often exhibit brightness variation, or shading. Of interest in computer vision is the inverse problem of how object shape may be recovered from such an image. This is referred to as the shape-from-shading problem. When the imaging conditions are such that an overhead point-source illuminates a smooth Lambertian surface, the problem may be formulated mathematically as that of finding a solution to an eikonal equation. In this paper, we seek images for which there are no corresponding object shapes. We are therefore concerned with the nonexistence of (bounded) solutions to certain eikonal equations. Specifically, we give a necessary and sufficient condition for a circularly-symmetric eikonal equation to admit exclusively unbounded solutions. In addition, we give a sufficient condition for an eikonal equation to have no solution. Examples are presented that elucidate the significance of these results for computer vision.
\end{abstract}

1. Introduction. A monochrome photograph of a smooth object will typically exhibit brightness variation, or shading. Of interest to researchers in computer vision has been the inverse problem of how object shape may be extracted from image shading. This shape-from-shading problem has been shown by Horn ([5]; see also [8, pp. 123-172]) to correspond to that of solving a first-order partial differential equation.

Received July 13, 1990.

1991 Mathematics Subject Classification. Primary 35F20, 68U10.

Key words and phrases. Shape from shading, Lambertian surface, eikonal equation, nonexistence of solutions.

(C) 1992 Brown University 
Specifically, one seeks a function $u(x, y)$, representing surface depth in the direction of the $z$-axis, satisfying the image irradiance equation

$$
R\left(u_{x}, u_{y}\right)=E(x, y)
$$

over $\Omega$. Here $R$ is a known function (the so-called reflectance map) capturing the illumination and surface reflecting conditions, $E$ is an image formed by (orthographic) projection of light along the $z$-axis onto a plane parallel to the $x y$-plane, and $\Omega$ is the image domain. In this formulation, it is implicitly assumed that

- A small surface portion reflects light independently of its position in space. Thus, scene radiance emitted in a given direction is dependent only on the illumination, the light-scattering properties of the surface material, and the surface normal. By implication, light sources are infinitely far away, and internal surface reflections are disallowed.

- Image irradiance is equal to the projected scene radiance.

Subsequent to the elucidation of the problem's fundamental form, much effort has been expended on generating computational techniques for solving the image irradiance equation (consult, for example, the annotated bibliography in Horn and Brooks [8]). In contrast with this, relatively little attention has been paid to the underlying theoretical issues.

Some progress has been made in the area of uniqueness and ambiguity of solutions. Given an image of some particular shape, the natural question arises as to whether it could also be the image of other shapes. Bruss ([2]; see also [8, pp. 69-88]), Deift and Sylvester [3], Brooks [1] and others have contributed important results in this area. Almost completely ignored, however, has been the issue of the existence of solutions.

Clearly, for any given shading problem, boundary conditions can readily be specified (for example, by constraining surface normals at various points) so as to ensure that there exists no solution. But, if a shading problem is such that there are no explicit boundary conditions, the reflectance map is known, and the image is smooth, can it be that no smooth surface shape could have generated the image? It was Horn [6] who first posed this problem and who coined the term impossible shading for a brightness pattern that could not be the image of a smooth surface.

In this article, we present two different classes of images for which there are no genuine shapes. Initially, we reveal a class of images for which only unbounded (and therefore physically-unrealisable) shapes exist. Next, we present a class of images exhibiting shading for which neither bounded nor unbounded shapes exist. This portion of the paper will refine an approach due to Horn, Szeliski, and Yuille [7]. Our entire treatment will apply to a reflectance map having special form, and this we shall now describe.

2. The eikonal equation. Assume that an overhead, distant point-source illuminates a Lambertian surface. A small portion of such a surface acts as a perfect diffuser, appearing equally bright from all directions. At first, this might seem to imply that Lambertian surfaces cannot exhibit other than constant shading. However, a curved object will, in general, receive illumination that differs in strength across the surface 
due to surface foreshortening, and it is this that will be responsible for change in image brightness.

Let a small surface portion with normal direction $\left(-u_{x},-u_{y}, 1\right)$ be illuminated by a distant, overhead point-source of unit power in direction $(0,0,1)$. The emitted radiance, as prescribed by Lambert's law, is given by the cosine of the angle between the two directions, namely $\left(u_{x}^{2}+u_{y}^{2}+1\right)^{-1 / 2}$. Since image irradiance is assumed to be identical in value with scene radiance, the corresponding image $E(x, y)$ satisfies

$$
\left(u_{x}^{2}+u_{y}^{2}+1\right)^{-1 / 2}=E(x, y) \text {, }
$$

which is the image irradiance equation appropriate for a Lambertian surface illuminated by a distant, overhead point-source. Noting that $0<E(x, y) \leq 1$, we may rewrite the above equation as

$$
u_{x}^{2}+u_{y}^{2}=\mathscr{E}(x, y),
$$

where $\mathscr{E}(x, y)$ is a transformed image given by $\mathscr{E}(x, y)=(E(x, y))^{-2}-1$. This eikonal equation has been studied extensively in connection with optical wavefront analysis and the Hamilton-Jacobi method of integrating particle motion equations. It was, furthermore, the basis for the uniqueness results of Bruss and others mentioned earlier. Our approach will be to consider forms of $\mathscr{E}(x, y)$ for which Eq. (1) has either exclusively unbounded solutions, or no solutions at all. We will therefore seek images that cannot arise from any physically-realisable Lambertian surfaces illuminated by a distant, overhead point-source.

3. Images without bounded solution. Let $R$ be either a positive number or $+\infty$. Let $f$ be a nonnegative continuous function on the interval $[0, R)$ vanishing exactly at zero. Let $D(R)$ denote the open disc in the $x y$-plane of radius $R$ centred at the origin. In this section we shall consider Eq. (1) over $D(R)$ with $\mathscr{E}$ given by

$$
\mathscr{E}(x, y)=f\left(\sqrt{x^{2}+y^{2}}\right) .
$$

With this special form of $\mathscr{E}$, the class of circularly-symmetric solutions is readily determined. Each solution in this class takes the form $\pm U+$ const, where

$$
U(x, y)=\int_{0}^{\sqrt{x^{2}+y^{2}}} \sqrt{f(\sigma)} d \sigma .
$$

Note that it is critical that $f$ vanish at zero so as to ensure the differentiability of $U$ at the origin of the $x y$-plane. Our eikonal equation may also admit noncircularlysymmetric solutions. The function $u(x, y)=x y$ provides an example of such a solution when $f(r)=r^{2}$ and $R=+\infty$. Unlike the class of circularly-symmetric solutions, the class of all noncircularly-symmetric solutions is not easily specified.

The principal aim of this section is to explore under what condition on $f$ all solutions to the corresponding eikonal equation are unbounded. Clearly, in the class of circularly-symmetric solutions the desired sufficient (and necessary) condition reads

$$
\int_{0}^{R} \sqrt{f(\sigma)} d \sigma=+\infty
$$


It is less evident, though true, that the same condition is sufficient in the general case. We have the following:

THEOREM 1. Let $f$ be a nonnegative continuous function on $[0, R)$ vanishing exactly at zero and satisfying (3). Then there is no bounded $C^{1}$ solution in $D(R)$ to Eq. (1) with $\mathscr{E}$ given by (2).

Proof. Let $u$ be a $C^{1}$ solution in $D(R)$ to Eq. (1) with $\mathscr{E}$ given by (2). Fix arbitrarily $\left(x_{0}, y_{0}\right)$ in $D(R) \backslash\{(0,0)\}$. Let $(x(t), y(t))$ be a solution of the Cauchy problem

$$
\begin{aligned}
\dot{x}(t) & =u_{x}(x(t), y(t)), \\
\dot{y}(t) & =u_{y}(x(t), y(t)), \\
x(0) & =x_{0}, \\
y(0) & =y_{0},
\end{aligned}
$$

with $t$ running over a maximal interval $\left(w_{-}, w_{+}\right)$. (Note that since we assume only continuity of $u_{x}$ and $u_{y}$, such a solution may not be unique.) Let $\Omega^{+}\left(\Omega^{-}\right)$ be the set of all points $p$ in the $x y$-plane such that there exists a sequence $\left(t_{n}\right)$ in $\left(w_{-}, w_{+}\right)$with $\lim _{n \rightarrow \infty} t_{n}=w_{+}\left(\lim _{n \rightarrow \infty} t_{n}=w_{-}\right)$and $\lim _{n \rightarrow \infty}\left(x\left(t_{n}\right), y\left(t_{n}\right)\right)=p$. We first show that at least one of the sets $\Omega^{+}$and $\Omega^{-}$fails to be a compact subset of $D(R)$.

Suppose, on the contrary, that both $\Omega^{+}$and $\Omega^{-}$are compact subsets of $D(R)$. Then clearly, $w_{ \pm}= \pm \infty$ (cf. [4, Theorem II 3.1]). Moreover, in virtue of the Poincaré-Bendixon theorem (cf. [4, Theorem VII 4.3]), both $\Omega^{+}$and $\Omega^{-}$contain either a periodic integral curve of the vector field $\left(u_{x}, u_{y}\right)$ or a singular point of this field. As we shall see shortly, periodic integral curves cannot exist. Since, in view of (1) and (2), the only singular point of $\left(u_{x}, u_{y}\right)$ is $(0,0)$, it will follow that both $\Omega^{+}$ and $\Omega^{-}$contain $(0,0)$.

Suppose that $(X(t), Y(t))$ is a periodic solution of the system

$$
\begin{aligned}
\dot{X}(t) & =u_{x}(X(t), Y(t)), \\
\dot{Y}(t) & =u_{y}(X(t), Y(t)),
\end{aligned}
$$

with period $\tau>0$. As $(X(t), Y(t))$ does not reduce to a singleton, there exists $0<t_{0}<\tau$ such that $\left(\dot{X}\left(t_{0}\right)\right)^{2}+\left(\dot{Y}\left(t_{0}\right)\right)^{2}>0$. If $0<\delta<\tau-t_{0}$, then

$$
\begin{aligned}
u(X(\tau-\delta), Y(\tau-\delta))-u(X(0), Y(0)) \\
\quad=\int_{0}^{\tau-\delta}\left[u_{x}(X(s), Y(s)) \cdot \dot{X}(s)+u_{y}(X(s), Y(s)) \cdot \dot{Y}(s)\right] d s \\
\quad=\int_{0}^{\tau-\delta}\left[(\dot{X}(s))^{2}+(\dot{Y}(s))^{2}\right] d s \\
\quad \geq \int_{0}^{t_{0}}\left[(\dot{X}(s))^{2}+(\dot{Y}(s))^{2}\right] d s,
\end{aligned}
$$

where the last integral is positive and independent of $\delta$. On the other hand, by the 
periodicity of $(X(t), Y(t))$,

$$
\lim _{\delta \rightarrow 0} u(X(\tau-\delta), Y(\tau-\delta))=u(X(\tau), Y(\tau))=u(X(0), Y(0)) .
$$

This contradiction shows that the periodic curve $(X(t), Y(t))$ defined above cannot exist.

Since $(0,0)$ is in both $\Omega^{+}$and $\Omega^{-}$, there exist two sequences $\left(t_{n}^{+}\right)$and $\left(t_{n}^{-}\right)$such that

$$
\lim _{n \rightarrow \infty} t_{n}^{ \pm}= \pm \infty
$$

and

$$
\lim _{n \rightarrow \infty} x\left(t_{n}^{ \pm}\right)=\lim _{n \rightarrow \infty} y\left(t_{n}^{ \pm}\right)=0 .
$$

Passing through $\left(x_{0}, y_{0}\right) \neq(0,0)$, the curve $(x(t), y(t))$ does not reduce to a singleton, and hence $(\dot{x}(T))^{2}+(\dot{y}(T))^{2}>0$ for some $T$. Choose a positive integer $n_{0}$ so that $\left|t_{n}^{ \pm}\right|>|T|$ for $n>n_{0}$. Then, for $n>n_{0}$,

$$
\begin{aligned}
& u\left(x\left(t_{n}^{+}\right), y\left(t_{n}^{+}\right)\right)-u\left(x\left(t_{n}^{-}\right), y\left(t_{n}^{-}\right)\right) \\
& \quad=\int_{t_{n}^{-}}^{t_{n}^{+}}\left[u_{x}(x(s), y(s)) \cdot \dot{x}(s)+u_{y}(x(s), y(s)) \cdot \dot{y}(s)\right] d s \\
& \quad=\int_{t_{n}^{-}}^{t_{n}^{+}}\left[(\dot{x}(s))^{2}+(\dot{y}(s))^{2}\right] d s \\
& \quad \geq \int_{-|T|}^{|T|}\left[(\dot{x}(s))^{2}+(\dot{y}(s))^{2}\right] d s,
\end{aligned}
$$

where the last integral is positive and independent of $n$. On the other hand, in view of (5),

$$
\lim _{n \rightarrow \infty} u\left(x\left(t_{n}^{ \pm}\right), y\left(t_{n}^{ \pm}\right)\right)=u(0,0) .
$$

This contradiction shows that at least one of the sets $\Omega^{+}$and $\Omega^{-}$fails to be a compact subset of $D(R)$.

Without loss of generality, we may assume that $\Omega^{+}$is not a compact subset of $D(R)$. Then there exists a sequence $\left(\tau_{n}\right)$ in $\left(0, w_{+}\right)$such that

$$
\lim _{n \rightarrow \infty}\left(x^{2}\left(\tau_{n}\right)+y^{2}\left(\tau_{n}\right)\right)=R^{2} \text {. }
$$

Set

$$
r(t)=\sqrt{x^{2}(t)+y^{2}(t)}
$$

and let $\theta$ be a real $C^{1}$ function such that

$$
\begin{aligned}
& x(t)=r(t) \cos \theta(t), \\
& y(t)=r(t) \sin \theta(t) .
\end{aligned}
$$


(In view of the continuity of $(x(t), y(t)), \theta$ is unique up to a multiple of $2 \pi$.) Then, for each $n$,

$$
\begin{aligned}
u\left(x\left(\tau_{n}\right), y\left(\tau_{n}\right)\right)-u\left(x_{0}, y_{0}\right) \\
\quad=\int_{0}^{\tau_{n}}\left[u_{x}(x(s), y(s)) \cdot \dot{x}(s)+u_{y}(x(s), y(s)) \cdot \dot{y}(s)\right] d s \\
\quad=\int_{0}^{\tau_{n}} \sqrt{u_{x}^{2}(x(s), y(s))+u_{y}^{2}(x(s), y(s))} \sqrt{(\dot{x}(s))^{2}+(\dot{y}(s))^{2}} d s \\
\quad=\int_{0}^{\tau_{n}} \sqrt{f(r(s))} \sqrt{(\dot{r}(s))^{2}+(\dot{\theta}(s))^{2} r^{2}(s)} d s \\
\quad \geq \int_{0}^{\tau_{n}} \sqrt{f(r(s))}|\dot{r}(s)| d s \\
\geq \int_{r(0)}^{r\left(\tau_{n}\right)} \sqrt{f(\sigma)} d \sigma .
\end{aligned}
$$

Since, in view of (3) and (6),

$$
\lim _{n \rightarrow \infty} \int_{r(0)}^{r\left(\tau_{n}\right)} \sqrt{f(\sigma)} d \sigma=+\infty
$$

we see that

$$
\lim _{n \rightarrow \infty} u\left(x\left(\tau_{n}\right), y\left(\tau_{n}\right)\right)=+\infty
$$

The proof is complete.

Interestingly, condition (3) is not only sufficient but also necessary for the unboundedness of all solutions to the equation in question. We have the following converse to Theorem 1:

THEOREM 2 . Let $f$ be a nonnegative continuous function in $[0, R)$ vanishing exactly at zero and satisfying

$$
\int_{0}^{R} \sqrt{f(\sigma)} d \sigma<+\infty
$$

Then every solution in $D(R)$ to Eq. (1) with $\mathscr{E}$ given by (2) is bounded. Moreover, if $u$ is any such solution, then

$$
\sup _{(x, y) \in D(R)} u(x, y)-\inf _{(x, y) \in D(R)} u(x, y) \leq 2 \int_{0}^{R} \sqrt{f(\sigma)} d \sigma .
$$

Proof. For any $(x, y)$ in $D(R) \backslash\{(0,0)\}$, let $(r, \theta)$ be the point in $(0, R) \times$ $[0,2 \pi)$ such that

$$
x=r \cos \theta, \quad y=r \sin \theta .
$$

Let $u$ be a solution in $D(R)$ to Eq. (1) with $\mathscr{E}$ given by (2). Set

$$
v(r, \theta)=u(x, y) .
$$

It is easily verified that $v$ satisfies the equation

$$
v_{r}^{2}+\frac{v_{\theta}^{2}}{r^{2}}=f(r)
$$




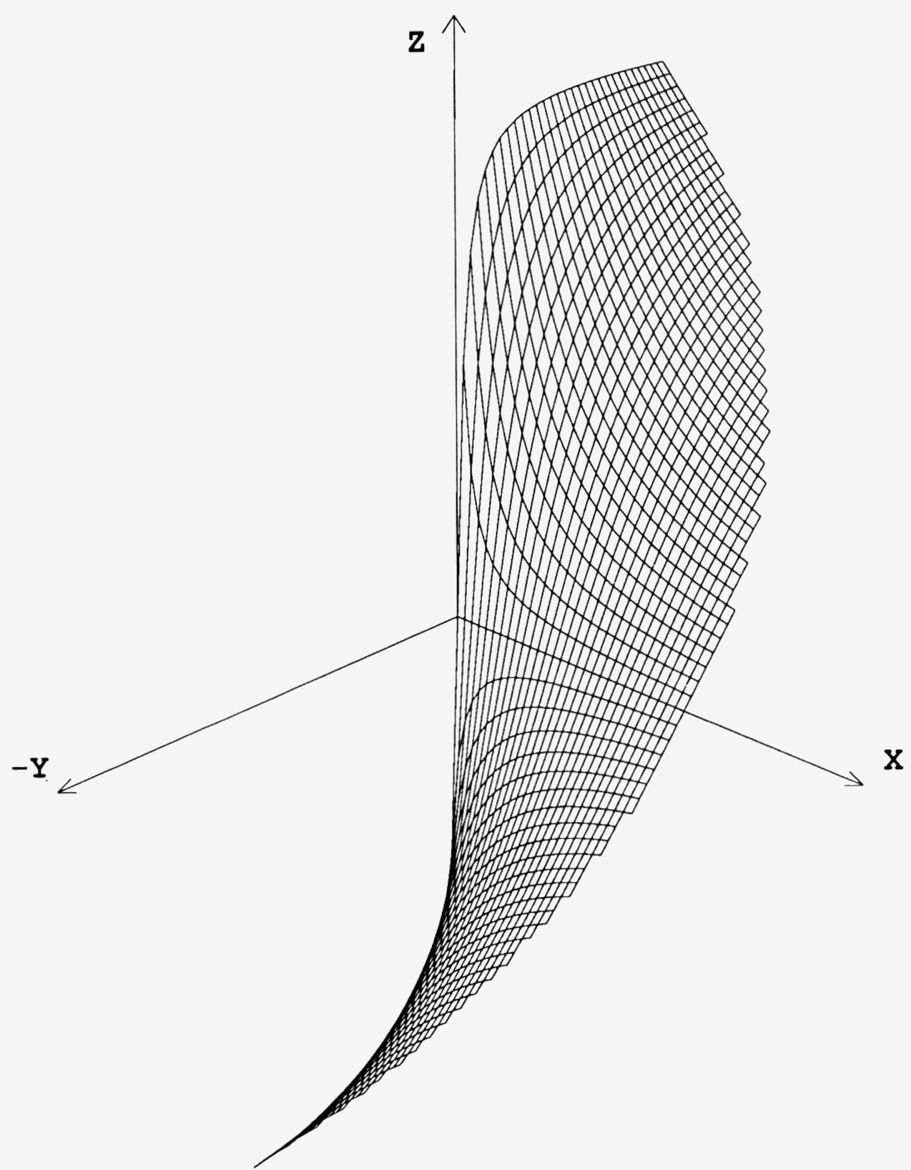

FIG. 1a. The graph of the function $\arctan \left(y x^{-1}\right)$ over $x>0, x^{2}+$ $y^{2}<1$.

It thus follows that for any $\sigma$ and any $\theta$

$$
\left|v_{r}(\sigma, \theta)\right| \leq \sqrt{f(\sigma)} .
$$

If $0<r^{\prime}<r$, then

$$
\begin{aligned}
\left|v(r, \theta)-v\left(r^{\prime}, \theta\right)\right| & \leq \int_{r^{\prime}}^{r}\left|v_{r}(\sigma, \theta)\right| d \sigma \\
& \leq \int_{r^{\prime}}^{r} \sqrt{f(\sigma)} d \sigma \\
& \leq \int_{0}^{R} \sqrt{f(\sigma)} d \sigma .
\end{aligned}
$$

Hence, by letting $r^{\prime}$ tend to zero,

$$
|u(x, y)-u(0,0)| \leq \int_{0}^{R} \sqrt{f(\sigma)} d \sigma,
$$




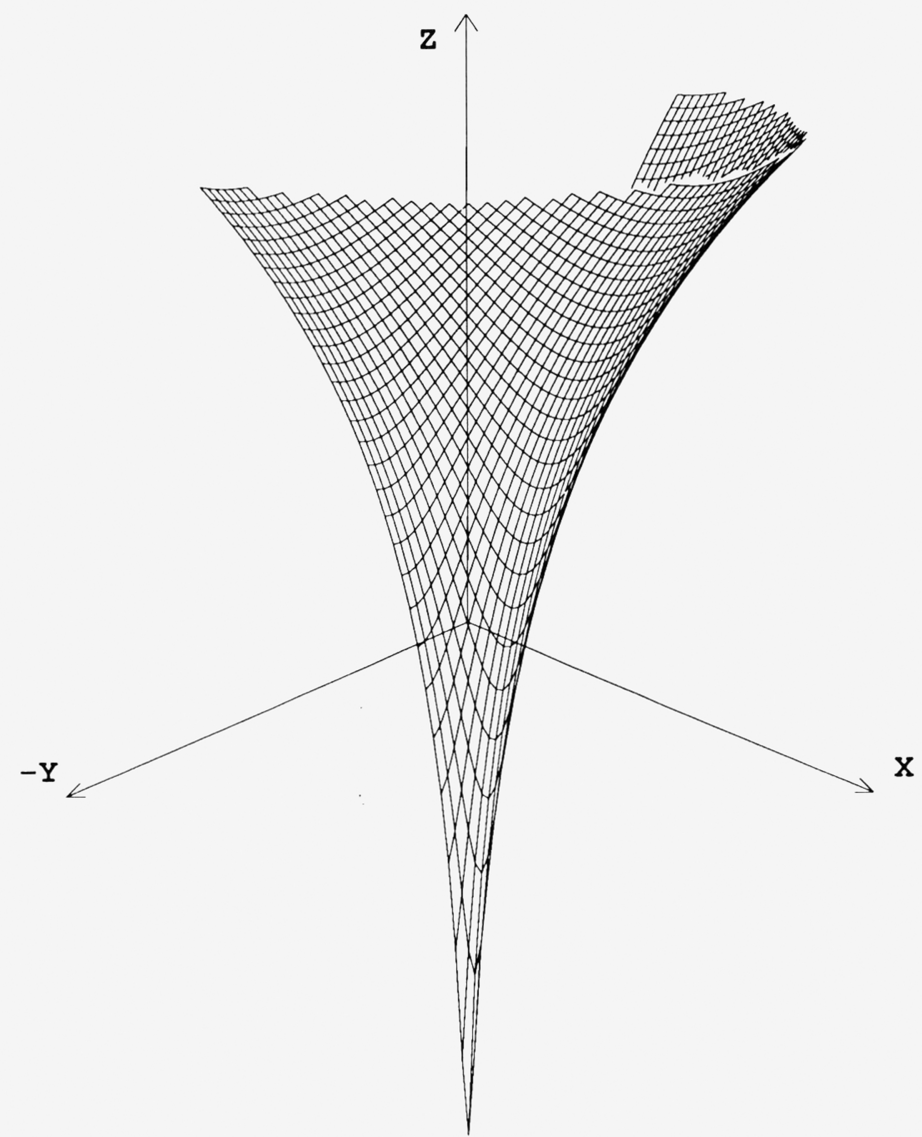

FIG. 1b. The graph of the function $\ln \left(x^{2}+y^{2}\right)^{1 / 2}+1$ over $x>$ $0, x^{2}+y^{2}<1$.

which implies that $u$ is bounded and, moreover, that

$$
\sup _{(x, y) \in D(R)} u(x, y)-\inf _{(x, y) \in D(R)} u(x, y) \leq 2 \int_{0}^{R} \sqrt{f(\sigma)} d \sigma .
$$

The proof is complete.

Observe that whether the integral $\int_{0}^{R} \sqrt{f(\sigma)} d \sigma$ is finite or infinite depends exclusively on the behaviour of $f$ near $R$. The integral will be infinite if, for example, $f(r)$ diverges to infinity sufficiently rapidly as $r$ tends to $R$. This means that, in the context of real images of Lambertian surfaces illuminated by an overhead point-source, a circularly-symmetric image cannot be derived from a genuine shape if it gets dark too quickly as the image boundary is approached. Note also that the above integral may be finite or infinite under the condition that $R$ is finite and $\lim _{r \rightarrow R} f(r)=+\infty$, which implies that the Euclidean norm of the gradient of any solution to (1) diverges to infinity as the circumference of $D(R)$ is approached. This is of interest in computer vision in that it relates to the notion of occluding boundary. The following examples show that the integral may be finite or infinite with the above condition being met: if $R=\pi / 2$ and $f(r)=\tan ^{2} r$, then the integral 
is infinite, and so no bounded solutions to (1) can exist; on the other hand, if $R=1$ and $f(r)=r^{2}\left(1-r^{2}\right)^{-1}$ (the image of the unit hemisphere centered at the origin), then the integral is finite, and so all solutions to (1) must be bounded.

Comparison of Theorems 1 and 2 reveals the following remarkable dichotomy: either all solutions to Eq. (1) with $\mathscr{E}$ given by (2) are bounded, or all solutions are unbounded, according to whether the integral $\int_{0}^{R} \sqrt{f(\sigma)} d \sigma$ is finite or infinite, respectively. The question then naturally arises as to whether there is an eikonal equation having both an unbounded and a bounded solution. This is answered in the affirmative when we note that, in the semidisc $\left\{(x, y) \in \mathbb{R}^{2}: x^{2}+y^{2}<1, x>0\right\}$, the bounded function $\arctan \left(y x^{-1}\right)$ and the unbounded function $\ln \sqrt{x^{2}+y^{2}}+1$ both satisfy the eikonal equation $u_{x}^{2}+u_{y}^{2}=\left(x^{2}+y^{2}\right)^{-1}$. The graphs of these functions are displayed in Figs. 1a (on p. 33) and 1 b (on p. 34), respectively.

4. Images without solution. We now establish the existence of transformed images $\mathscr{E}$ for which there is no solution to Eq. (1). The theorem presented below is a refinement of that due to Horn, Szeliski, and Yuille [7]; its proof given below elaborates an outline also due to Horn et al.

TheOREM 3. Let $\Omega$ be a bounded domain in the $x y$-plane with boundary $\partial \Omega$ being a piecewise $C^{1}$ curve of length $\ell_{\partial \Omega}$. Let $\left(x_{0}, y_{0}\right)$ be a point in $\Omega$ and $r$ be a positive number such that the closed disc $\bar{D}\left(x_{0}, y_{0}, r\right)$ of radius $r$ centered at $\left(x_{0}, y_{0}\right)$ is contained in $\Omega$. Suppose $\mathscr{E}$ is a nonnegative continuous function on the closure of $\Omega$, positive in $\Omega$, such that

$$
4 r \sqrt{\mathscr{E}_{1}}>\ell_{\partial \Omega} \sqrt{\mathscr{E}_{2}}
$$

where $\mathscr{E}_{1}=\min \left\{\mathscr{E}(x, y):(x, y) \in \bar{D}\left(x_{0}, y_{0}, r\right)\right\}$ and

$$
\mathscr{E}_{2}=\max \{\mathscr{E}(x, y):(x, y) \in \partial \Omega\} .
$$

Then there is no $C^{1}$ solution to Eq. (1) in $\Omega$.

Proof. Let $K$ be a compact subset of $\Omega$, with $C^{1}$ boundary, containing $\bar{D}\left(x_{0}, y_{0}, r\right)$; let $u$ be a $C^{1}$ solution to (1) in $\Omega$; and let $(x(t), y(t))$ be a solution of the Cauchy problem (4) defined on a maximal interval $\left(w_{-}, w_{+}\right)$. We claim that there exists $t_{-}$and $t_{+}$in $\left(w_{-}, w_{+}\right)$such that $t_{-}<0<t_{+}$and $P_{ \pm}^{+}=\left(x\left(t_{ \pm}\right), y\left(t_{ \pm}\right)\right)$ belong to $\partial K$.

Suppose, for example, that no such $t_{+}$exists. Then, clearly, $w_{+}=+\infty$ and all points $(x(t), y(t))$ with $t>0$ lie in $K$. Retaining the notation from the proof of Theorem 1, we apply the Poincaré-Bendixon theorem to ascertain that $\Omega^{+}$contains either a periodic integral curve of the vector field $\left(u_{x}, u_{y}\right)$ or a singular point of this field. Since $\mathscr{E}$ is positive in $\Omega$, it follows that $\left(u_{x}, u_{y}\right)$ has no singular points. On the other hand, periodic curves are excluded by the same argument as in the proof of Theorem 1. This contradiction establishes the claim.

Let $t_{-}<\tau_{-}<0$ and $0<\tau_{+}<t_{+}$be such that $Q_{ \pm}=\left(x\left(\tau_{ \pm}\right), y\left(\tau_{ \pm}\right)\right)$belong to 
the circumference of $\bar{D}\left(x_{0}, y_{0}, r\right)$. We have

$$
\begin{aligned}
u\left(P_{+}\right)-u\left(P_{-}\right) & =\int_{t_{-}}^{t_{+}}\left[u_{x}(x(s), y(s)) \cdot \dot{x}(s)+u_{y}(x(s), y(s)) \cdot \dot{y}(s)\right] d s \\
& =\int_{t_{-}}^{t_{+}}\left[(\dot{x}(s))^{2}+(\dot{y}(s))^{2}\right] d s \\
& \geq \int_{\tau_{-}}^{\tau_{+}}\left[(\dot{x}(s))^{2}+(\dot{y}(s))^{2}\right] d s \\
& =\int_{\tau_{-}}^{\tau_{+}} \sqrt{\mathscr{E}(x(s), y(s))} \sqrt{(\dot{x}(s))^{2}+(\dot{y}(s))^{2}} d s \\
& \geq \sqrt{\mathscr{E}_{1}} \int_{\tau_{-}}^{\tau_{+}} \sqrt{\left.(\dot{x}(s))^{2}+\dot{y}(s)\right)^{2}} d s .
\end{aligned}
$$

Of course, $\int_{\tau_{-}}^{\tau_{+}} \sqrt{(\dot{x}(s))^{2}+(\dot{y}(s))^{2}} d s$ is equal to the length of the segment of the curve $(x(t), y(t))$ between $Q_{-}$and $Q_{+}$and as such is no smaller than $2 r$. Hence

$$
u\left(P_{+}\right)-u\left(P_{-}\right) \geq \sqrt{\mathscr{E}_{1}} 2 r .
$$

Let $(X(t), Y(t))$ be the shorter of the two curves joining $P_{-}$and $P_{+}$along $\partial K$, parametrized by $t$ running over $\left(T_{-}, T_{+}\right)$, with $P_{ \pm}=\left(X\left(T_{ \pm}\right), Y\left(T_{ \pm}\right)\right)$. Let

$$
\mathscr{E}_{\partial K}=\max \{\mathscr{E}(x, y):(x, y) \in \partial K\} \text {. }
$$

Using the Cauchy-Schwarz inequality, we get

$$
\begin{aligned}
u\left(P_{+}\right)-u\left(P_{-}\right) & =\int_{T_{-}}^{T_{+}}\left[u_{x}(X(s), Y(s)) \cdot \dot{X}(s)+u_{y}(X(s), Y(s)) \cdot \dot{Y}(s)\right] d s \\
& \leq \int_{T_{-}}^{T_{+}} \sqrt{u_{x}^{2}(X(s), Y(s))+u_{y}^{2}(X(s), Y(s))} \sqrt{(\dot{X}(s))^{2}+(\dot{Y}(s))^{2}} d s \\
& \leq \sqrt{\mathscr{E}_{\partial K}} \int_{T_{-}}^{T_{+}} \sqrt{(\dot{X}(s))^{2}+(\dot{Y}(s))^{2}} d s \\
& \leq \frac{1}{2} \sqrt{\mathscr{E}_{\partial K}} \ell_{\partial K} .
\end{aligned}
$$

Hence, in view of $(8)$,

$$
4 r \sqrt{\mathscr{E}_{1}} \leq \ell_{\partial K} \sqrt{\mathscr{E}_{\partial K}}
$$

Since $K$ can be chosen so that $\ell_{\partial K} \sqrt{\mathscr{E}_{\partial K}}$ is arbitrarily close to $\ell_{\partial \Omega} \sqrt{\mathscr{E}_{2}}$, we obtain a contradiction with (7).

The proof is complete.

Note that the theorem proved is of local character: if $\Omega$ is a subset of a domain $\Delta$ and $\mathscr{E}$ is a nonnegative function on $\Delta$ whose restriction to $\Omega$ satisfies (7) for some choice of $\bar{D}\left(x_{0}, y_{0}, r\right)$ in $\Omega$, then, obviously, there is no $C^{1}$ solution to (1) in $\Delta$. Reformulated in terms of Lambertian shading, this locality property can be expressed as saying that no genuine image can admit too dark a spot on too bright a background, 


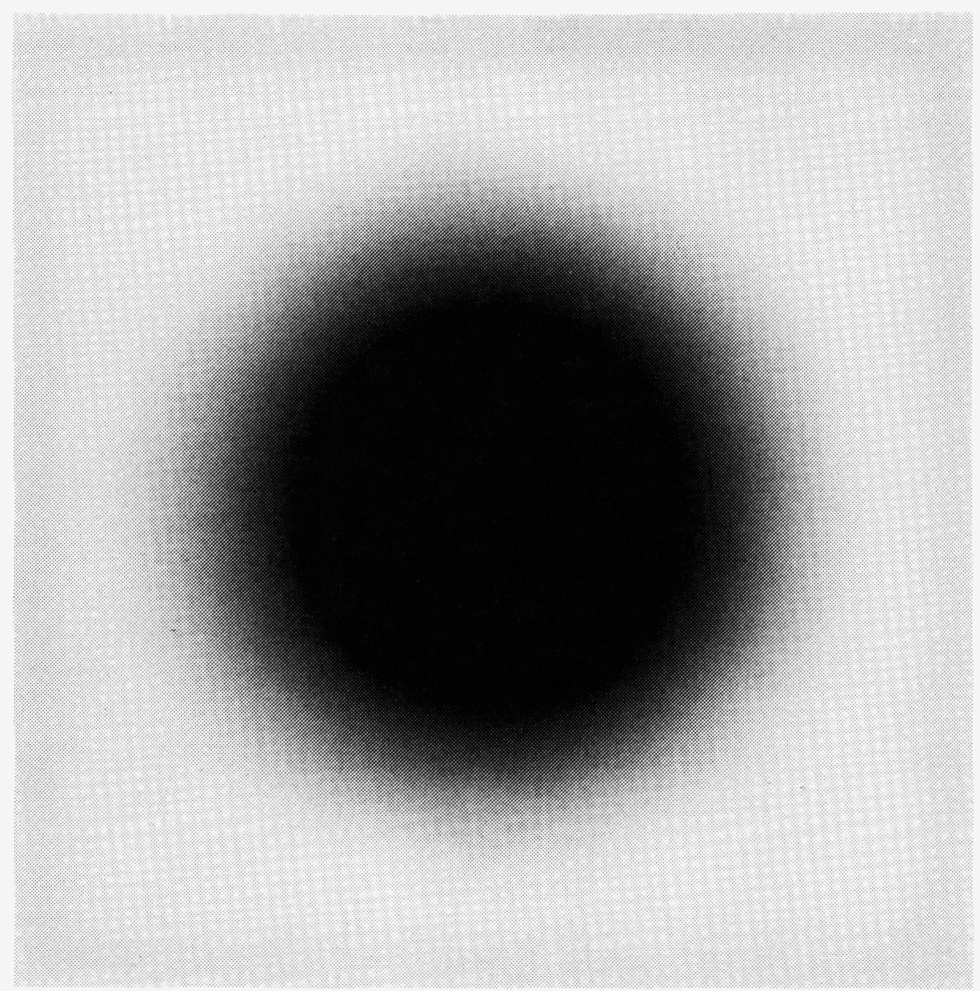

FIG. 2. Shading without shape.

assuming that the background does not contain a point having unit brightness. The precise balance between the qualifications "too dark" and "too bright" is, of course, given by condition (7). An example of shading without shape is given in Fig. 2.

Further insight may be gained by considering the following. Suppose that a planar rubber sheet is inclined slightly away from the horizontal, and that a coin is glued to the underside of the sheet. Imagine twisting the coin so as to make a portion of the sheet more steeply inclined (see Fig. 3a on p. 38). An image of the sheet will now exhibit a dark area surrounded by a bright background. This area may be made arbitrarily dark by a further twisting of the coin, while the background may be brightened by having the sheet inclined more closely to the horizontal. We therefore appear to be in a position to formulate a contradiction to Theorem 3. However, in attempting to generate a specific counter-example in this way, it soon becomes apparent that the image of the steep area cannot be made sufficiently dark and large without the surface exhibiting at least one stationary point where $u_{x}=u_{y}=0$ (see Fig. $3 \mathrm{~b}$ on p. 38). Such a point would result in a violation of the condition in Theorem 3 that $\mathscr{E}>0$ in $\Omega$. The theorem therefore survives intact. 


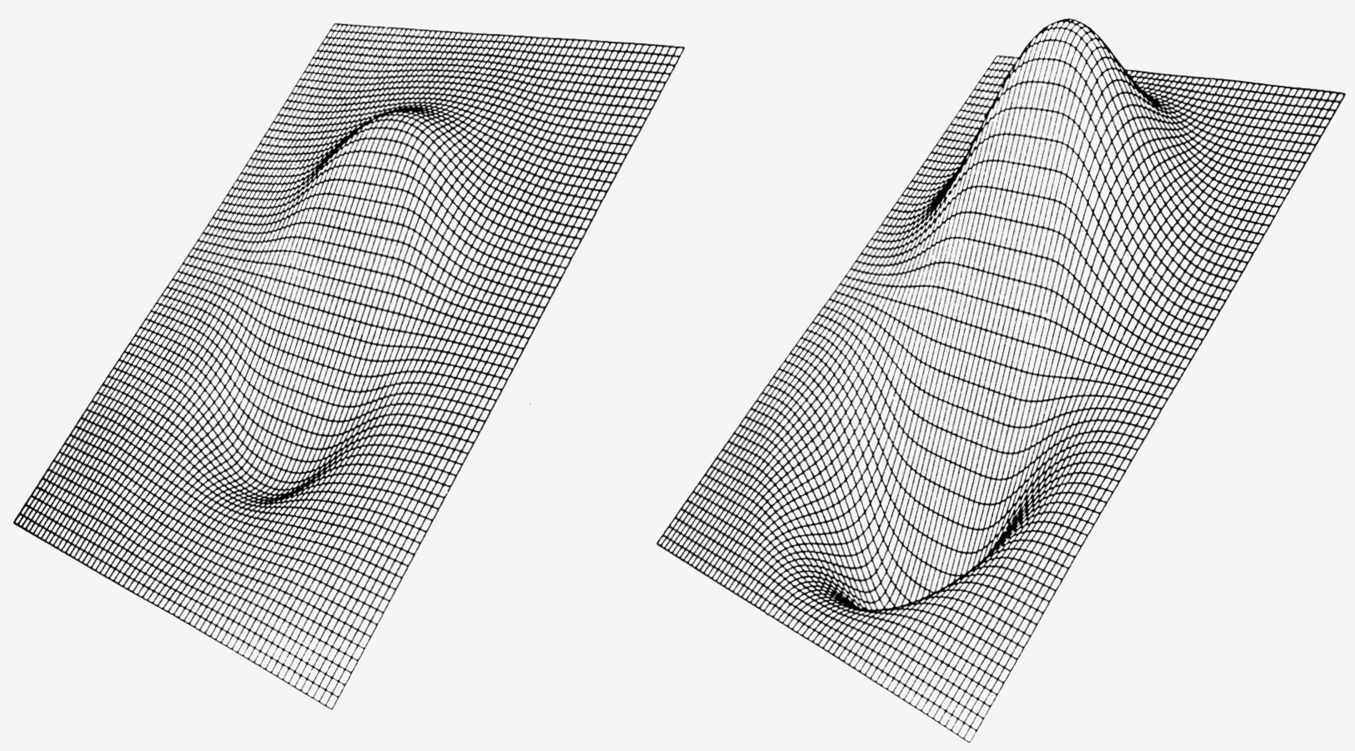

FIG. 3a. Surface without stationary point. FIG. 3b. Surface with a stationary point.

Acknowledgments. W. Chojnacki expresses gratitude to Flinders University for the opportunity to spend time as a visiting researcher, and to Warsaw University for granting leave. The authors thank Professor B. Horn for his posing of a number of stimulating problems in this area. Additionally, the authors gratefully acknowledge the assistance of D. Gibbins in the generation of figures.

\section{REFERENCES}

[1] M. J. Brooks, Two results concerning ambiguity in shape from shading, Proceedings of the National Conference on Artificial Intelligence, Washington, D.C., August 22-26, 1983, The American Association for Artificial Intelligence, sponsor, pp. 36-39

[2] A. R. Bruss, The eikonal equation: some results applicable to computer vision, J. Math. Phys. 23, 890-896 (1982)

[3] P. Deift and J. Sylvester, Some remarks on the shape-from-shading problem in computer vision, J. Math. Anal. Appl. 84, 235-248 (1981)

[4] P. Hartman, Ordinary Differential Equations, Wiley, New York, 1973

[5] B. K. P. Horn, Obtaining shape from shading information, The Psychology of Computer Vision, P. H. Winston, ed., McGraw-Hill, New York, 1975

[6] B. K. P. Horn, private communication, 1987

[7] B. K. P. Horn, R. Szeliski, and A. Yuille, private communication, 1989

[8] B. K. P. Horn and M. J. Brooks, eds., Shape from Shading, MIT Press, Cambridge, Mass., 1989 